\title{
Article
}

\section{A New Class of Autopoietic and Cognitive Machines}

\author{
Rao Mikkilineni ${ }^{1, *}$
}

1 Ageno School of Business, Golden Gate University, San Francisco, CA 94105, USA; rmikkilineni@ggu.edu;
* Correspondence: $\underline{\text { rmikkilineni@ggu.edu ; }}$

\begin{abstract}
Making computing machines mimic living organisms has captured the imagination of many since the dawn of digital computers. However, today's artificial intelligence technologies fall short in replicating even the basic autopoietic and cognitive behaviors found in primitive biological systems. According Charles Darwin, the difference in mind between humans and the higher animals, great as it is, certainly is one of degree and not of kind. Autopoiesis refers to the behavior of a system that replicates itself and maintains its own identity and stability while facing fluctuations caused by external influences. Cognitive behaviors model the system's state, sense internal and external changes, analyze, predict and take action to mitigate any risk to its functional fulfilment. How did intelligence evolve? what is the relationship between the mind and body? Answers to these questions should guide us to infuse autopoietic and cognitive behaviors into digital machines. In this paper we use recent advances in our understanding of general theory of information, and the role of structures in managing the transformations between information and knowledge to pave the path to infuse autopoietic and cognitive functions into digital computing and build a new class of intelligent machines going beyond the current state of the art.
\end{abstract}

Keywords: Cognition, Computing Models, Deep Learning, Autopoiesis, Knowledge Structures, Structural Machines, Autopoietic Machines.

\section{Introduction}

Physical and mental structures which transform information and knowledge are the essential ingredients of intelligence. In most living organisms, genes encode the "life" processes and pass them on from the survivor to the successor. The genetic knowledge structures include executable workflows and control processes that describe stable patterns to optimally utilize the resources available to assure the organism's creation and safe keeping in interacting with its environment. Creation involves the processes that use knowledge to transform matter and energy. The system with "self" awareness is assembled using physical structures with several constituent components. Safe keeping involves the ability to dynamically monitor and control organism's own behavior along with its interactions with its environment using the genetic descriptions. Intelligent systems augment inherited knowledge through genes with cognitive processes embedded in the nervous systems and neural networks. The system uses its components to gain information through its sensory components and converts it to knowledge using its neural network. The neurons that fire together wire together to capture the knowledge about the events that caused it and the neurons that are wired together fire together to exhibit the autopoietic and cognitive behaviors. The neural network mechanisms are used to develop a history through memorizing the transactions and identifying new associations through their analysis. In short, the genetic computing model consisting of complex multi-networks of genes combined with neural network processing allows the formulation of descriptions and execution of workflow components with not only the content of how to accomplish a task but also provide the context, constraints, control and communication to assure systemic coordination to accomplish the overall purpose of the system embedded in the genome. 
Intelligent systems also have developed internal and external communication structures that allow sentient behavior (the ability to sense and react to change the circumstances without disruption of current workflow execution both within the system and in its interactions with its environment). Computing (the ability to transform information obtained through the senses, create and process knowledge structures capturing the dynamics), communication (the ability to pass information within its own components and with external systems) and cognition (the ability to create and execute processes that sense and react to changing circumstances) are essential ingredients of intelligence that provide sentience and resilience (the ability to the ability to know and adapt appropriately to changing circumstances).

The biological structures are described as complex adaptive systems (CAS) composed of many interrelated and interacting components (made up of components which exploit the properties of atoms, molecules, compounds etc., to create the composed structures). CAS [1] exhibits self-organization, non-linearity, transition between states of order and chaos and emergence. The system often exhibits behavior that is difficult to explain through an analysis of the systems' constituent parts. Such behavior is called emergent. CAS are complex systems that have the capacity to adapt to their environment through an evolution-like process and are isomorphic to networks (nodes linked by edges). The system evolves into a complex multi-layer network and the functions of the nodes and the composed structure defines the global behavior of the system as whole. Sentience, resilience and intelligence are the result of these structural transformations and dynamics exhibiting the autopoietic and cognitive behaviors.

In this paper, we discuss the evolution of sentience, resilience and intelligence in living organisms using information processing structures and describe a theoretical model with their essential characteristics which are autopoiesis and cognition. This model then allows us to design ways to infuse autopoietic and cognitive behaviors into digital information processing structures built using digital automata. In section 2, we describe the lessons from studying the evolution of autopoietic and cognitive behaviors in living organisms. In section 3, we present a theoretical model based on general theory of information and the theory of structures to model the structures exhibiting the autopoietic and cognitive behaviors. In section 4, we describe recent attempts to use this model to infuse autopoietic and cognitive behaviors in digital information processing structures to improve their sentience, resilience and intelligence going beyond the current state. In section 5 , we conclude with some observations on this approach and its impact on current and future state of information technologies.

\section{Evolution of Sentience, Resilience and Intelligence in Living Organisms}

\subsection{Intelligence and Natural Evolution}

An important question is - how did living organisms evolve from being mere physical and chemical structures to develop the complex behaviors of autopoiesis and cognition we observe in all living beings with varying degrees of sentience, resilience and intelligence? Experts [2] tell us that "In the microbial world, decisions are made by monitoring the current state of the system, by processing this information and by taking action with the ability to take into account several factors such as recent history, the likely future conditions and the cost and benefit of making a particular decision. At the population level, microbes are also capable of hedging their bets, by having individuals of an isogenic population in different states even when experiencing the same environmental conditions, and they are also able to make collective decisions that cause the entire population to respond in a particular way. Microbes are able to make decisions based on different criteria of information and also to perform the decision-making using different mechanisms, utilizing different types of molecular networks."

As Darwin said [3] in the conclusion of his 'long argument', "And as natural selection works solely by and for the good of each being, all corporeal and mental endowments will 
tend to progress towards perfection". He also said "Natura non facit saltum" or nature does not make jumps.

Indeed, it seems that the nature chose "punctuated equilibria" for the evolution with increasing levels of sentience resilience and intelligence. According to Westerhoff [2], "Microbes exhibit similar characteristics of intelligence as higher organisms and humans, such as decision making, robust adaptation, association and anticipation, self-awareness and problem solving capabilities." Living organisms persist by virtue of complex interactions among many components organized into dynamic, environment-responsive networks that span multiple scales and dimensions. These studies show that the evolution of biological systems from the underlying physical and chemical structures was a gradual transformation of independent component structures interacting with each other and behaving like a complex adaptive system. The system's evolution based on individual component structure and function and their interaction with each other and the external environment is the result of emergent properties of complex adaptive system. As fluctuations in their interaction and scale of the components increased, the emergent property allowed the formation of complex multi-layer networks with behaviors that are different from any of the individual components.

Let us go back to the question, what did the living organisms evolve from and how? Our current scientific theories point to the structural evolution from physical and chemical components aided by random fluctuations in their interactions among themselves and with their environment. Function, structure and fluctuations play a key role in the evolution of physical and chemical structures obeying the laws of transformation governing matter and energy. The laws of thermodynamics influence the microscopic and macroscopic behaviors of these structures. According to the first law of thermodynamics, if the energy of the system consisting of structures that are interacting with each other is conserved, it would reach equilibrium and the structures tend to be stable. The second law of thermodynamics states that if a closed system is left to itself, it tends to increase disorder and entropy which is a measure of the disorder. However, if the system is able to exchange energy with the environment outside, it can increase its order by decreasing entropy inside and transferring it to the outside. This allows the structures to use energy from outside and form more complex structures with lower entropy or higher order.

These concepts have been the foundation for the theories of phase transitions in physics [4] (as Prigogine mentioned in his Nobel lecture, "nonequilibrium may be a source of order"), and the theory of complex adaptive systems which has been applied to understand the economic behaviors of groups engaged in commerce [1] ("In the complex adaptive system of the economy, understanding the micro-level behaviors of individuals is essential to understanding how the system as a whole behaves"). Living beings have, through evolution and natural selection, perfected the art of increasing order inside by exchanging energy and entropy with the outside.

Five "Big Ideas" that distinguish Complex adaptive systems are [1] as follows:

1. They are open, dynamic, and nonlinear systems, which are far from equilibrium.

2. Individual components interact with each other and their environment without a global view of information. Local dynamics and behaviors are completely dependent on energy and entropy considerations and the energy exchange.

3. The system dynamics often, results in the formation of networks where each component interacts with other components and could be modeled as nodes with specific functional behavior (exploiting matter and energy transformations), exchanging information with other components based on energy and entropy considerations; networks of relationships change over time.

4. As fluctuations cause local variations, their scale and magnitude determine the degree of disequilibrium of the system and the system exhibits emergence where the global behavior of the system is completely unpredictable based on the behaviors of individual components.

5. The evolutionary process of differentiation, selection, and amplification provides the system with novelty and is responsible for its growth in order and complexity. 
It seems that the living organisms have evolved from the soup of physical and chemical structures [2] in three different phases where the characteristics of the evolving systems are different. In all the phases, the evolution involves complex multi-layer networks. According to Westerhoff et al., [2] "Biological networks constitute a type of information and communication technology (ICT): they receive information from the outside and inside of cells, integrate and interpret this information, and then activate a response. Biological networks enable molecules within cells, and even cells themselves, to communicate with each other and their environment."

\subsubsection{Phase1: Metabolic Networks:}

In a cell or microorganism, the processes that generate matter, energy, information transfer, and the cell fate specification are seamlessly integrated through a complex network of various cellular constituents and reactions [5]. Biologists and theoreticians [6] have analyzed several organisms and shown that, despite significant variances in their individual constituents and pathways, metabolic networks display the same topologic scaling properties demonstrating striking similarities to the inherent organization of complex non-biological systems. They conclude that the metabolic organization is not only identical for all organisms, but complies with the design principles of robust and errortolerant scale-free networks, and may represent a common blueprint for the large scale organization of interactions among al cellular constituents. The scale-free networks are characterized by their degree of distribution which is a Poisson distribution whereas a random network is characterized by a Gaussian distribution. The degree of a node in a network is the number of connections a node has to other nodes and the degree distribution is the probability distribution of these degrees over the whole network. Scale-free networks contribute to a high degree of error tolerance or resilience; that is, the ability of their nodes to communicate is unaffected by the failure of some randomly chosen nodes.

The lesson here is that the networked nature of the complex adaptive system and its dynamics contribute to the properties of sentience, resilience and some form of intelligence and is observed even in primitive forms of life.

\subsubsection{Phase 2: Interacting Networks}

With the advent of proteins, networks of proteins have evolved based on their binding properties. In the interacting network model, the proteins are nodes and two nodes are connected by a nondirected edge if the two proteins bind [7, 8]. Physical interactions dictate the architecture of the cell in terms of how direct associations between molecules constitute protein complexes, signal transduction pathways and other cellular machinery. Genetic interactions define functional relationships between genes, giving insight into how this physical architecture translates into phenotype. Replication, signaling and composition are essential characteristics required for autopoietic and cognitive processes and they are exhibited in the interactive networks of genes.

\subsubsection{Phase 3: Neural Networks}

In order to address the questions "How does the brain work?" and "How can we build intelligent machines?" [9], we should look to our understanding from genomics [10], neuroscience [11,12,13] and information science [14 - 20] all of which are throwing new light. Figure 1 shows our current understanding of how the mind, brain and body function based on both theoretical and experimental findings presented by various scholars. On the left hand side of figure 1, the genes and neurons are depicted. On the right hand side, the reptilian complex which uses both the brain and body to sense information, convert into knowledge and capture the cognitive behaviors. 
From Reptilian Brain to Mammalian Brain

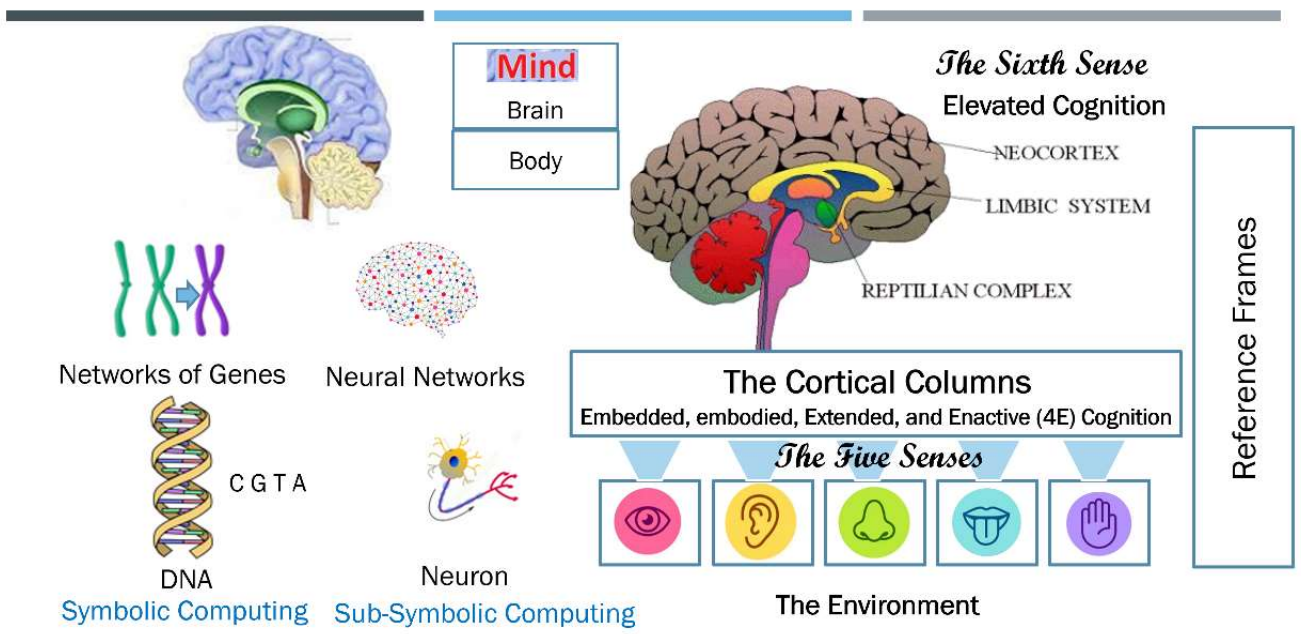

PHILOSOPHY AND COMPUTING CONFERENCE - IS4SI SUMMIT 2021

Figure 1: Two views of the mind, brain and body components.

The five senses are used by the reptilian cortical columns to provide embedded, embodied, extended and enactive (4E) cognitive behaviors. The reference frames [1] provide a knowledge representation in the form of neural networks where the nodes that are fired together based on the inputs from the cortical columns wire together and the nodes that are wired together fire together to exhibit the behaviors using the body to interact with the environment. The knowledge representation captures the relationships between various entities that constitute the system and the entities that the system interacts with. The relationships along with their dynamic behaviors are represented in the form of neural networks. The networks of genes provide the autopoietic behaviors using the various physical structures that constitutes the body and the brain. The neural networks provide the mechanism for sensing, converting the information into a common knowledge representation across the five senses using the old reptilian brain. The new brain provides higher level information processing using the common knowledge representation to enhance the $4 \mathrm{E}$ cognition from the five senses with a sixth sense of elevated cognition. It is important to note that the genes use physical and chemical processes converting matter and energy to transform components (exhibiting autopoietic behaviors) using symbolic computing that involves DNA and RNA where the symbols are characterized by the 4 elements of DNA. The information in DNA is stored as a code made up of four chemical bases: adenine $(A)$, guanine $(G)$, cytosine $(C)$, and thymine $(T)$. Human DNA consists of about 3 billion bases, and more than 99 percent of those bases are the same in all people.

On the other hand, the neural networks in the brain, a typical neuron collect signals from others through a host of fine structures called dendrites. The neuron sends out spikes of electrical activity through the axon (the output and conducting structure) which can split into thousands of branches. The signals excite the neurons and the neurons that fire together wire together to provide the mechanism for converting information into knowledge, which in turn is used in elevated cognition and exhibiting cognitive behaviors. This is known as sub-symbolic computing. While the reptilian brain provides subsymbolic computing based information, the neocortex uses it to process higher level functions and uses the network of genes to make appropriate actions to maintain stability within the system and interact with the outside based on its knowledge maintained in the neural networks.

Armed with this knowledge, we can now look for a model to capture the mind, brain and body and their relationships to matter, energy, information and knowledge which enable the autopoietic and cognitive behaviors. 


\subsection{Human Intelligence, the Mind-Body Debate and the Theory of Structural Reality}

Since the time of ancient Greek philosopher Plato (420s-340s BCE) introduced the Ideas/Forms, the mind-body problem which asks the question - what is the relationship between mind and body? - has been the subject of debate until recently [16, 17]. With the new scientific interpretation of Plato Ideas/Forms in the form of physical and mental structures supplemented by various findings in neuroscience and genomics, we have a new insight into how the mind and body interact. In simple terms, Plato's Theory of Forms asserts that the physical world is not really the 'real' world; instead, ultimate reality exists beyond our physical world. The physical world is the material stuff we see and interact with on a daily basis; this physical world is always changing and imperfect. The world of Ideas and Forms, however, exists beyond the physical world and exists in the form of ideal structures as illustrated in figure 2.

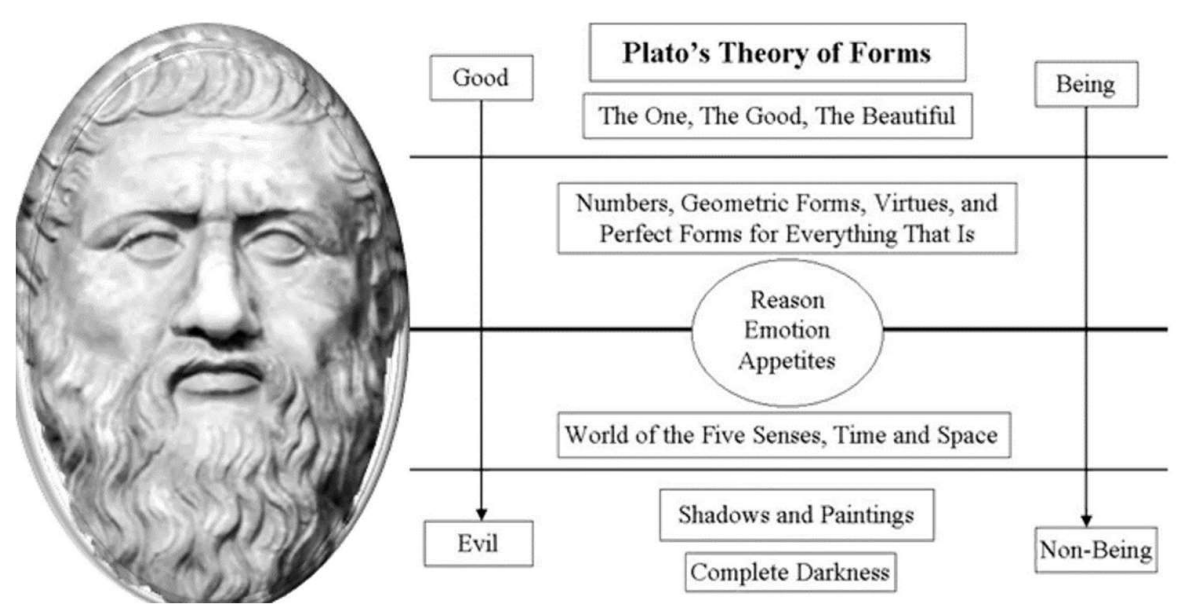

Figure 2: Plato Ideas/Forms

The general theory of information (GTI) [16] and the theory of structural machines [17] dealing with transformation of information and knowledge tell us that information per se belongs to the ideal world of structures, which is the scientific realization of the world of Plato - Ideas or Forms. However, it comes to and functions, in the physical world of living beings by acquiring physical representations and physical carriers. Living beings and the machines invented by them, both work with these carriers and physical representations to access, process, use and store information. On the other hand, the physical universe is made up of structures which interact with each other using the laws of transformation dealing with matter and energy. These interactions result in more complex physical structures. All physical structures contain information, which has the capacity to change the states of these structures (the concept of from being to becoming).

However, life forms have evolved to use physical structures in the form of genes and neurons to create mental models of physical structures as abstract structures. Unlike physical structures, mental structures only exist in the abstract models created by living organisms using their own physical structures. The mental energy required to create mental structures is derived from their physical structures. GTI provides means for the abstract mental model representations using ideal structures that exist in the ideal world. Abstract structures deal with information and knowledge and knowledge to information is as matter is to energy. It provides the tools to transform information into knowledge and follow the dynamics of these mental structures. These are the tools in the form of fundamental triads, named sets, knowledge structures, cognizing oracles and the structural machines. These tools and their use are also discussed in various books and publications. In the next section, we summarize key lessons from GTI and present the Burgin-Mikkilineni thesis [18] which provides a model not only to describe the super-symbolic, symbolic, and sub- 
symbolic computations in living organisms but also a model [19 - 21] to infuse autopoietic and cognitive behaviors into digital automata.

\section{General Theory of Information and the Burgin-Mikkilineni Thesis (BMT)}

GTI [15] provides a unified context for existing directions in information studies, making it possible to elaborate on a comprehensive definition of information; explain relations between information, data, and knowledge; and demonstrate how different mathematical models of information and information processes are related. We briefly summarize the tools that GTI provides to model information processing structures and their behaviors both in humans and digital machines.

Information unit is described by the existence or non-existence ( 1 or 0$)$ of an entity or an on object that is physically observed or mentally conceived. The difference between an entity and an object is that the entity is an abstract concept with attributes such as a computer with memory and CPU. An object is an instance of an entity with an identity, defined by two components which are the object-state and object-behavior. An attribute is a key value pair with an identity (name) and a value associated with it. The attribute state is defined by its value. Information is related to knowledge and is defined by the relationships between various entities and their interactions (behaviors) when the values of the attributes change. A named set is a fundamental triad that defines the knowledge about two different entities (Figure 3).

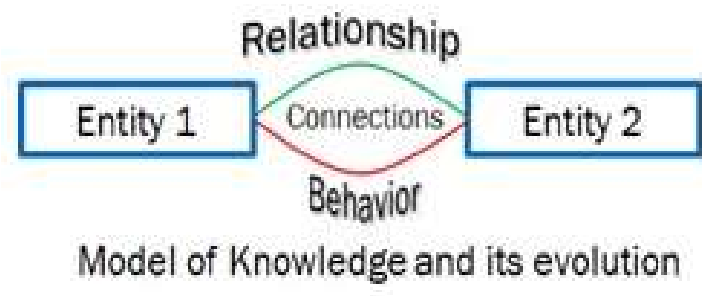

Figure 3: The fundamental triad as a knowledge structure derived from information

A knowledge structure [ defines various triadic relationships between all the entities that are contained in a system. The knowledge structure provides the schema and various operations to evolve the schema from one state to another. Various instances of the knowledge structure schema are used to model the domain knowledge and process information changes as they evolve with changes in their entities and their attributes and behaviors.

The structural machine [19-21] is an information processing structure that represents the knowledge structures as schema and performs operations on them to evolve information changes in the system from one instant to another when any of the attributes of any of the objects change.

The structural machines supersede the Turing machines by their representations of knowledge and the operations that process information [20]. Triadic structural machines with multiple general and mission-oriented processors enable autopoietic behaviors.

The Burgin-Mikkilineni thesis states that "The ontological BM thesis states that the autopoietic and cognitive behavior of artificial systems must function on three levels of information processing systems and be based on triadic automata. The axiological BM thesis states that the efficient autopoietic and cognitive behavior has to employ structural machines." A genome in the language of GTI) encapsulates "knowledge structures" coded in the form of DNA and executed using the "structural machines in the form of genes and neurons which use physical and chemical processes (dealing with conversion of matter and energy). The information accumulated through biological evolution is encoded into knowledge to create the genome which contains the knowledge network defining the function, structure and the autopoietic and cognitive processes to build and evolve the system while managing both deterministic and non-deterministic fluctuations in the interactions among the internal components or their interactions with the environment. 
GTI tells us that information is represented, processed and communicated using physical structures. The physical universe, as we know it, is made up of structures that deal with matter and energy. As Mark Burgin [15] points out energy and matter are different but intrinsically connected with one another. Matter cannot exist without energy (at least, zero energy), while energy is always contained in physical bodies. The BM thesis also states that "the knowledge about the genome and the GTI allow us to postulate a thesis that allows us to design a new class of digital automata by infusing autopoietic and cognitive behaviors."

A digital genome is defined as a collection of "knowledge structures" coded in executable form to be processed with "structural machines" implemented using digital genes (in the form of symbolic computing algorithms) and digital neurons (in the form of subsymbolic neural net algorithms) both of which use stored program control implementation of Turing machines. "The digital genome enables digital process execution to discover the computing resources in the environment, use them to assemble the hardware, cognitive apparatuses in the form of digital genes and digital neurons and evolve the process of sentient, resilient, intelligent and efficient management of both the self and the environment with embedded, embodied, enacted and extended (4E) cognitive processes.

The digital genome incorporates the knowledge in the form of hierarchical intelligence with a definition of the sentient digital computing structures that discover, monitor and evolve both the self and the interactions with each other and the environment based on best practices infused in them.

The digital genome specifies the execution of knowledge networks using both symbolic computing and sub-symbolic computing structures. The knowledge network consists of a supersymbolic network of symbolic and sub-symbolic networks executing the functions defined in their components. The structure provides the system behavior and evolution maintaining the system's stability in the face of fluctuations in both internal and external interactions. The digital genome encapsulates both autopoietic and cognitive behaviors of digital information processing structure capable sentience, resilience and intelligence. The digital genome typifies infused cognition as opposed to evolved cognition in biological systems. The infusion is made by the human operators who teach the machines on how to evolve. Self-learning and self-evolution with consciousness are not yet present in these digital structures."

In the next section, we will describe how to design a new class of autopoietic and cognitive machines using existing information technologies such as cloud computing, containers and their management tools just as the neocortex overlay utilized existing reptilian cognitive behaviors.

\section{Infusing Autopoietic and Cognitive Behaviors into Digital Automata}

Figure 4 shows the new class of machines that integrate symbolic and sub-symbolic computing structures. Symbolic computing (using algorithms and operations on symbolic data structures) provides the equivalent of the networks of genes that interact with the physical resources using the transformation laws of matter and energy. The neural network algorithms provide the equivalent of extracting information and converting into knowledge with $4 \mathrm{E}$ cognition. Super-symbolic computing provides the mechanism to represent knowledge from multiple sources as a knowledge network where the nodes contain the knowledge structures representing the state of various entities, relationships and their behaviors as an always on executable service module. 


\section{New Structural Machine \& The Old Turing Machine}

\section{The Sixth Sense Elevated Cognition}

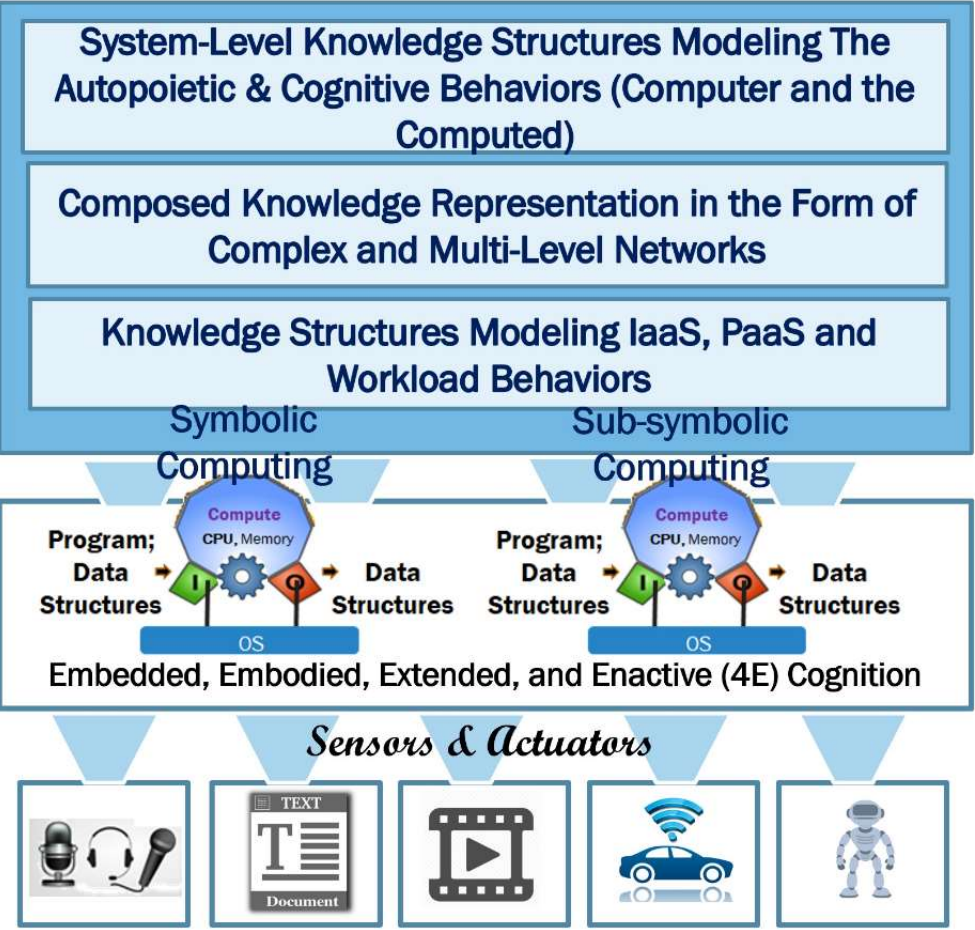

The Environment

Figure 4: Super-symbolic computing structure with a common knowledge representation using knowledge structures and structural machines

The inputs to the knowledge node provide the information that triggers the behavioral changes in the nodes that impact other node behaviors through communication of information as outputs from the nodes. All the knowledge nodes wired together fire together to execute a collective behavior. The knowledge network is implemented as a structural machine that provides operations on the Knowledge structure schema [20, 21]

A design of the implementation of a structural machine using knowledge structures that represent the "life processes" of a computational workflow application is shown in figure 5. The digital genome is like a cell that contains all the executables, their control structures and operational details about an application designed to be a distributed computing structure executed in infrastructures offered by different providers. It contains knowledge about what resources (CPU, Memory, storage, network etc.) are required for each component and where they are available and how to use them. It is, in essence, a specification of all "life processes" defined in the genome as knowledge structures (entities, relationships and behaviors). When the digital genome is executed in a computer as a program, it functions as a manager of downstream networks that will be created, monitored and managed based on the "life process" definitions for these downstream networks. It is done by creating downstream network manager which knows what their downstream functions (like specialized functional cells (such as a web server, application server and a data base) that execute specific functions and communicate with other cells influencing their behaviors. All specialized functions are defined as knowledge structures (again entities, relationships and behaviors executed using local CPU, Memory, OS, Database, file system, program executables etc. ). 


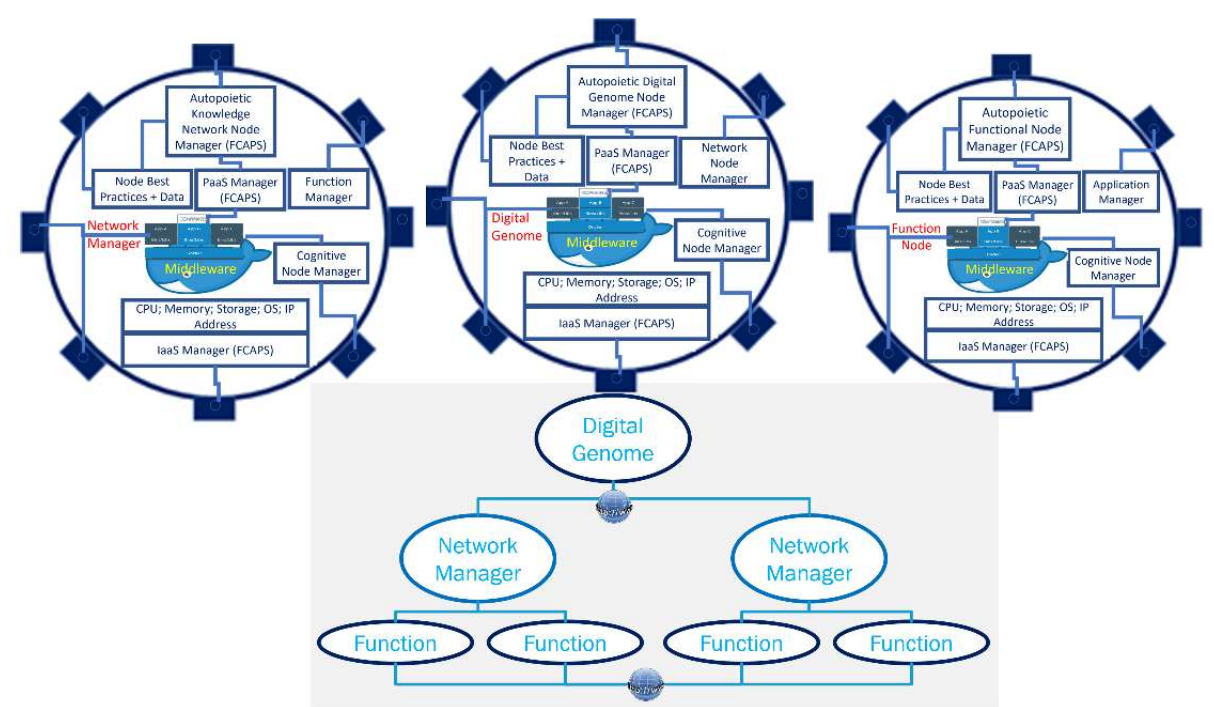

Figure 5: The design for implementing the digital genome and the knowledge network.

The figure shows a digital genome node executing in a cloud resource which configures, monitors and manages the knowledge network management nodes. Each network node in turn configure, execute, monitor and manage the various functional nodes that constitute functional knowledge network with entities, relationships and behaviors. The knowledge functional nodes wired together fire together to execute the collective behaviors. The autopoietic and cognitive behaviors that model the life processes are executed at each layer to maintain global stability and intended outcomes. Deviations are monitored at each level and corrections are made based on life process definitions at each level.

The unique differentiation from current state of the art is the introduction of common knowledge representation in the form of knowledge structures that combines the knowledge obtained from symbolic and sub-symbolic computations in the functional nodes. The structural machine implementation with triadic automata [19 -21] provides global stability of and global outcomes from the various components to meet the global intent while maintaining the local autonomy of individual component management. The super-symbolic overlay manages global optimization while dealing with the fluctuations in the interactions of components impacted by local constraints. The real-time global monitoring, and management provides the capability for optimizing global behavior using downstream knowledge network reconfiguration.

\section{Conclusions}

This paper brings together our learnings from the genomics, neuroscience and the science of information processing structures to show a new path to design and build a new class of autopoietic and cognitive machines that go beyond the current state of the art in making machines mimic living organisms. There is no new science here and we just use the knowledge gained from various philosophers, mathematicians and eminent thinkers from Plato to professor Burgin and suggest a possible new path to classical computer scientists and IT professionals who are pursuing with symbolic and sub-symbolic computing structures to mimic living beings. GTI and the theories of structural reality, while not widely recognized by the mainstream scholars, to date, provide a unified framework for modeling real structures with mental structures, reason about them and relate them to ideal structures described by Plato ideas/Forms. They also provide a framework to discover the relationship between computing, communication, cognition, consciousness and culture that permeate beyond digital automata [17]. As far the related work, we believe this approach is novel and we are not aware of any similar proposal except for the references provided in this paper. 
For those practitioners who insist on implementation and market acceptability as a proof for the validity of a theory, we invite active participation to prove or disprove the arguments presented in this paper.

Funding: "This research received no external funding"

Acknowledgments: Author wishes to thank prof. Mark Burgin for various in-depth discussions on general theory of information and the theory of structural machines. This paper is mainly a result of these discussions. Author wishes to express his gratitude to late Prof. Peter Wegner for many discussions that shaped his understanding of computer science. The author also expresses his gratitude for the education he received at the university of California, San Diego where many Nobel Laureates and other eminent scholars influenced his thinking. Prof. Walter Kohn (Nobel Laureate 1998) had a special influence as a thesis advisor and mentor.

Conflicts of Interest: “The authors declare no conflict of interest."

\section{References}

1. Beinhocker, E.D. The Origin of Wealth; Harvard Business Review Press, Kindle Edition; Boston, USA, 2006 ; p. 40.

2. Westerhoff Hans V., Brooks Aaron N., Simeonidis Evangelos, García-Contreras Rodolfo, He Fei, Boogerd Fred C., Jackson Victoria J., Goncharuk Valeri, Kolodkin Alexey. Macromolecular networks and intelligence in microorganisms, Frontiers in Microbiology, Volume 5, 2014; P. 5. https://www.frontiersin.org/article/10.3389/fmicb.2014.00379

3. Darwin, Charles, 1809-1882. On the Origin of Species by Means of Natural Selection, or Preservation of Favoured Races in the Struggle for Life. London :John Murray, 1859.

4. Prigogine, Ilya. “Time, Structure, and Fluctuations.” Science, vol. 201, no. 4358, American Association for the Advancement of Science, 1978, pp. 777-85, http://www.jstor.org/stable/1746122.

5. Hartwell, L.H., Hopfield, J.J., Leibler, S. \& Murray, A.W. From molecular to modular cell biology. Nature 402, C47-52 (1999).

6. Jeong H, Tombor B, Albert R, Oltvai Z N, Barabasi A L, The large-scale organization of metabolic networks, Nature,2000, 407:651-654

7. Réka Albert; Scale-free networks in cell biology. J Cell Sci 1 November 2005; 118 (21): $4947-4957$. doi: https://doi.org/10.1242/jcs.02714

8. Beyer A, Bandyopadhyay S, Ideker T. Integrating physical and genetic maps: from genomes to interaction networks. Nat Rev Genet. 2007;8(9):699-710. doi:10.1038/nrg2144

9. Arbib, M.A.; The Elements of Brain Theory and Neural Networks; In Book The Handbook of the Brain Theory and Neual Networks, 2nd ed.; Arbib, M.A., Eds.; The MIT Press, Cambridge, USA, 2003; pp. 12.

10. Itai Yanai, Lercher Martin, "The Society of Genes." Harvard University Press, 2016.

11. Dehaene, Stanislas. (2014). "Consciousness and the Brain: Deciphering How the Brain Codes Our Thoughts" Penguin Group, New York.

12. Dehaene, Stanislas. (2014). "Consciousness and the Brain: Deciphering How the Brain Codes Our Thoughts” Penguin Group, New York.

13. Damasio, A. (2010) Self comes to mind. Pantheon, a division of Random House, Inc., New York.Author 1, A.B.; Author 2, C. Title of Unpublished Work. Abbreviated Journal Name stage of publication (under review; accepted; in press).

14. Jeff Hawkins, (2021). "A Thousand Brains: A New Theory of Intelligence.” Basic Books, New York.

15. Burgin, M. Theory of Information: Fundamentality, Diversity and Unification, World Scientific: Singapore, 2010.

16. Burgin, M. Theory of Knowledge: Structures and Processes; World Scientific: New York, NY, USA; London, UK; Singapore, 2016.

17. Burgin, Mark. Structural Reality. New York: Nova Science Publishers, 2012.

18. Burgin, M. and Mikkilineni, R. On the Autopoietic and Cognitive Behavior, EasyChair Preprint no. 6261, version 2., 2021. https://easychair.org/publications/preprint/tkjk

19. Burgin, M. Triadic Automata and Machines as Information Transformers, Information, v. 11, No. 2, 2020, 102; doi:10.3390/info11020102

20. Burgin, M., Mikkilineni, R. and Phalke, V. Autopoietic Computing Systems and Triadic Automata: The Theory and Practice, Advances in Computer and Communications, v. 1, No. 1, 2020, pp. 16-35

21. Burgin, M. and Mikkilineni, R. From Data Processing to Knowledge Processing: Working with Operational Schemas by Autopoietic Machines, Big Data Cogn. Comput. 2021, v. 5, 13 (https://doi.org/10.3390/bdcc5010013). 